\title{
Multipactor in a Coaxial Line Under the Presence of an Axial DC Magnetic Field
}

\author{
D. González-Iglesias, A. M. Pérez, S. Anza, J. Vague, B. Gimeno, Member, IEEE, V. E. Boria, Senior Member, IEEE, \\ D. Raboso, C. Vicente, J. Gil, F. Caspers, Senior Member, IEEE, and L. Conde
}

\begin{abstract}
The main goal of this letter is the analysis of the multipactor effect within a coaxial waveguide structure when an external axial dc magnetic field is applied. We have designed and manufactured a coaxial waveguide sample that has been immersed within a long solenoid. Numerical and experimental results confirm a significant change in the $\mathrm{RF}$ breakdown behavior with regard to the case without the axial dc magnetic field, as well as the existence of single- and double-surface multipactor regimes. Good agreement between theory and experimental data has been found.
\end{abstract}

Index Terms-Coaxial transmission line, dc magnetic field, multipactor effect, RF breakdown.

\section{INTRODUCTION}

$\mathbf{M}$ ULTIPACTOR breakdown is a phenomenon that takes place on devices operating under vacuum conditions and high-power RF electromagnetic fields [1]. The multipactor effect is present in different environments such as satellite passive components used for space communications, klystrons, cyclotrons, and particle accelerators, where it is named electron cloud phenomenon [2]. The multipactor breakdown occurs when the trajectories of free electrons, existing inside the device, are synchronized with the RF electric field. This phenomenon produces an exponential growth of the electron population, generating several negative effects that degrade the device performance.

Multipactor phenomenon has been deeply studied for different kinds of waveguides. In particular, multipactor analysis in coaxial lines has attracted the interest of many researchers [3]-[7]. In this context, the presence of dc magnetic fields has been recently used for the partial or total discharge mitigation,

Manuscript received January 23, 2012; accepted January 31, 2012. Date of publication March 16, 2012; date of current version April 20, 2012. This work was supported in part by the Spanish Government through R\&D project TEC2010-21520-C04-01. The review of this letter was arranged by Editor E. A. Gutiérrez-D.

D. González-Iglesias, A. M. Pérez, J. Vague, and V. E. Boria are with the Departamento de Comunicaciones-iTEAM, Universidad Politécnica de Valencia, 46022 Valencia, Spain.

B. Gimeno is with the Departamento de Física Aplicada-ICMUV, Universidad de Valencia, 46100 Valencia, Spain (e-mail: benito.gimeno@uv.es).

S. Anza, C. Vicente, and J. Gil are with AURORASAT, 46022 Valencia, Spain.

D. Raboso is with the European Space Agency/European Space Research and Technology Centre, 2200 AG Noordwijk, The Netherlands.

F. Caspers is with CERN, 1211 Geneva, Switzerland.

L. Conde is with the Departamento de Física Aplicada, E.T.S.I. Aeronáuticos, Universidad Politécnica de Madrid, 28040 Madrid, Spain.

Color versions of one or more of the figures in this letter are available online at http://ieeexplore.ieee.org.

Digital Object Identifier 10.1109/LED.2012.2186952 as reported in [8], where the direction of the magnetic field remains in the transverse propagation plane of a coaxial geometry, and in [9] and [10], where an axial magnetic field is inserted within a rectangular waveguide for multipactor suppression.

In this letter, we have studied the effect of an axial dc magnetic field in the multipaction existing within a standard coaxial transmission line. In the first section, we discuss the theoretical model employed for simulations. Next, we have designed, manufactured, and tested a simple coaxial sample, as well as a long solenoid for dc magnetic field generation. An experimental test bed for multipactor detection has been developed. Finally, the comparison between experimental data and theory proves the existence of different multipactor regimes under the presence of an external dc magnetic field.

\section{THEORY}

The commercial FEST3D software [11] was used to perform the simulations of multipactor within the coaxial line. Indeed, this code is based on the 3-D tracking of a set of electrons governed by electric and magnetic external fields. To proceed, the Lorentz force equation is numerically solved by means of the leap-frog algorithm, as described in [12]. The considered fields are the superposition of the time-harmonic electric and magnetic coaxial TEM mode fields of frequency $f$, and the external axial uniform dc magnetic field $B_{\mathrm{DC}}$. Following the Monte Carlo technique, when an electron hits on the inner or the outer metallic walls, the code allows absorbing the electron or releasing secondary electrons depending on the impact conditions. This is done by using the secondary-electron-yield coefficient $\delta$ (SEY) [13] of the material. Thus, the SEY model for copper is described in [14] with a first crossover $W_{1}=$ $25 \mathrm{eV}, \delta_{\max }=2.25$, and an incident electron energy value for $\delta_{\max }$ given by $W_{\max }=175 \mathrm{eV}$. A Maxwellian distribution for computing the secondary electrons' velocities has been used, considering a mean of $4 \mathrm{eV}$ and a standard deviation of $2 \mathrm{eV}$. The multipactor onset criterion used by FEST3D is based on the detection of an exponential growth rate.

\section{EXPERIMENTAL SETUP}

The coaxial sample for multipactor test is shown in Fig. 1. The inner and outer radius dimensions are $a=1.515 \mathrm{~mm}$ and $b=3.490 \mathrm{~mm}$, respectively; the length of the total sample is $90.4 \mathrm{~mm}$. Both conductors were made of copper, and the existing gap between them is $d=b-a=1.975 \mathrm{~mm}$.

An external dc magnetic field is applied to the coaxial sample by means of a long solenoid, with dimensions selected to ensure 


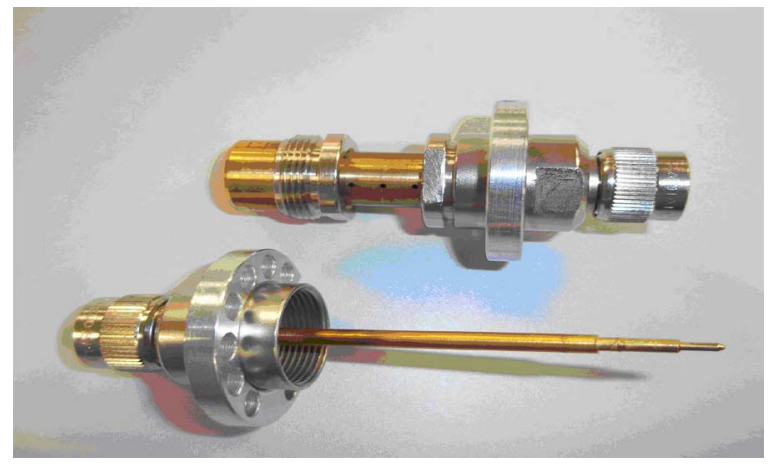

Fig. 1. Manufactured coaxial sample for multipactor test.

a uniform magnetic field in the central region, where the coaxial sample is inserted. According to these specifications, we have designed and manufactured a solenoid formed by around 8250 turns with $30-\mathrm{cm}$ length and $1.905-\mathrm{cm}$ radius, being its electrical resistance is $71.8 \Omega$. This solenoid was calibrated by means of a magnetic probe mounted in a translation linear stage, which moves along the axis of symmetry of the solenoid. The linear relation between the solenoid dc current $I_{\mathrm{DC}}$ and the magnetic field in the center of the solenoid gives $B_{\mathrm{DC}}=3.8 \mathrm{mT}$ when $I_{\mathrm{DC}}=100 \mathrm{~mA}$.

An experiment to validate FEST3D simulations was carried out at the European Space Agency Val Space Consortium Laboratory [15]; the experimental setup is the standard one, commonly employed in multipactor measurements (see [14]). The coaxial sample was placed centered inside the solenoid, and three detection methods were used, i.e., the measurement of nulling between the input and the reflected waves at the carrier frequency, the third-harmonic frequency detection of the transmitted signal, and the measurement of the secondary electrons generated in the discharge with an electron probe. During the measurements, a pressure vacuum chamber was in an interval $10^{-5}-10^{-7}$ mbar.

Measurements were performed at an RF frequency of $f=$ $1.145 \mathrm{GHz}$, which corresponds to a frequency gap of $f \times d=$ $2.261 \mathrm{GHz} \cdot \mathrm{mm}$. The RF signal generator operated in pulsed mode with a pulse width of $20 \mu$ s and a duty cycle of $2 \%$. An optical fiber connected to an ultraviolet mercury lamp $(254 \mathrm{~nm})$ was used to generate photoelectrons, which was inserted into the coaxial sample through a venting hole. Multipactor RF power threshold was measured for several dc magnetic field strengths. Both experimental and theoretical results are shown in Fig. 2.

\section{Discussion}

As we can see in Fig. 2, there is good agreement between experimental and computed points. Small differences can be related to the inaccuracy of the SEY model used in the theoretical simulations. According to both experiment and theory, the presence of the dc magnetic field has minimized and maximized the RF power threshold a factor of $84 \%$ and $23 \%$ in comparison to the zero magnetic field case, respectively.

In order to have a better understanding of the obtained results, electron trajectories have been represented for some significant points, which have been marked in Fig. 2 as A, B,

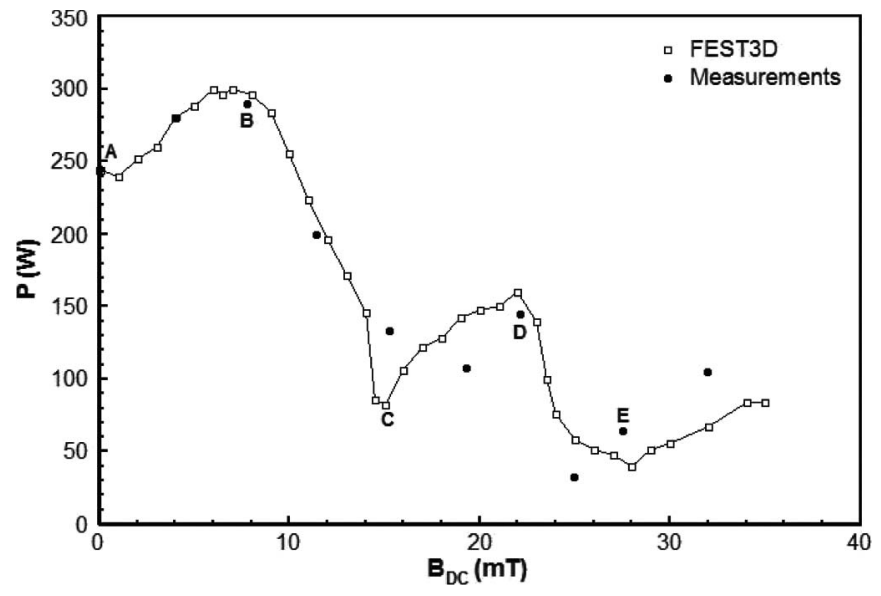

Fig. 2. Multipactor RF input power threshold as a function of the dc magnetic field strength. Experimental and theoretical results are shown. Experimental errors associated with both magnitudes have been estimated in $\pm 2 \mathrm{~W}$ and $\pm 1 \mathrm{mT}$, respectively.

TABLE I

Summary of the Results Showed IN Fig. 2

\begin{tabular}{|c|c|c|c|c|c|c|}
\hline Point & $B_{D C}(\mathrm{mT})$ & $P(\mathrm{~W})$ & $f_{c}(\mathrm{MHz})$ & $r_{L}(\mu m)$ & $S E Y_{i}$ & $S E Y_{o}$ \\
\hline A & 0 & 245 & 0 & $\infty$ & 2.0 & 1.1 \\
B & 8 & 290 & 216 & 875 & 2.9 & 2.9 \\
C & 15 & 86 & 420 & 449 & 1.2 & 0.5 \\
D & 22 & 145 & 620 & 305 & 0.4 & 1.1 \\
E & 27 & 65 & 770 & 245 & 2.2 & 0.9 \\
\hline
\end{tabular}

C, D, and E. In Table I, we have summarized the most relevant results, including cyclotron frequency $f_{c}$, Larmor radius $r_{L}$, and mean SEY value on the inner $i$ and the outer $o$ surfaces. For point $\mathrm{A}$, we find a hybrid double-surface multipactor mode of order 1 for the inner-to-outer conductor path and order 3 for the outer-to-inner case, as shown in Fig. 3. (Note that we define the multipactor order in terms of the number of RF semiperiods.) Due to the presence of the magnetic field, for point B, we have observed a mixing between double- and single-surface multipactor regimes. This effect is due to the increase in the $\mathrm{dc}$ magnetic field, which tends to bend the electron orbits around the magnetic field flux lines, as shown in Fig. 3.

For point $\mathrm{C}$, the dc magnetic field is strong enough to avoid electrons reaching the opposite conductor so that there are only single-surface orbits, as illustrated in Fig. 3. Indeed, electron trajectories are confined near the conductor where electrons are launched, but the discharge is generated only on the inner wall. Electrons starting around the central region of the gap and near the outer conductor impact on the outer wall with too low kinetic energy values to generate true secondaries; hence, they do not contribute to the discharge (see Table I). However, electrons starting from the inner conductor produce a single-sided multipactor regime of order 4 . The variation of the RF electric field of the TEM fundamental mode with $1 / r$ ( $r$ being the cylindrical radial coordinate) produces an asymmetric behavior in both inner and outer walls, providing more kinetic energy to the electrons emitted from the internal wall than those launched from the external one. Thus, when the RF power is increased above the threshold for the same value of magnetic field, we observe discharges on both surfaces, which enhance multipactor effect in comparison with point $\mathrm{C}$. 

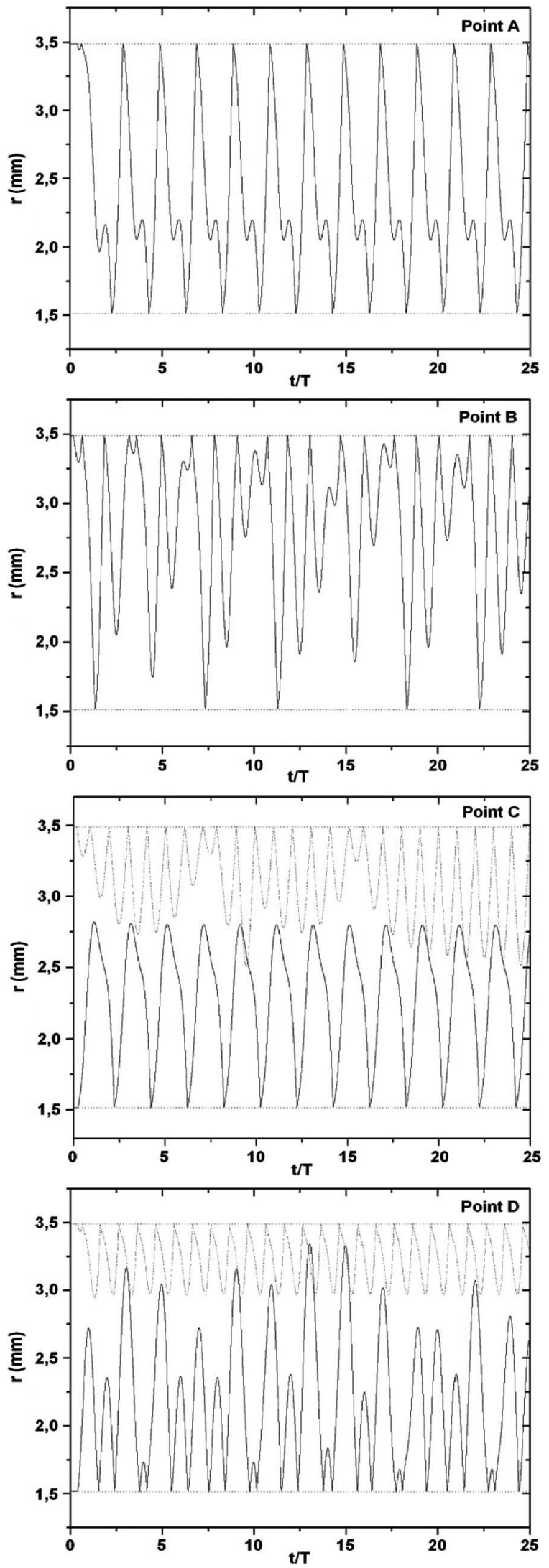

Fig. 3. Electron trajectories of points A, B, C, and D in Fig. 2. The cylindrical radial coordinate of the effective electron $r$ has been plotted as a function of the normalized time $t / T, T$ being the RF period $(T=1 / f)$. The inner and outer radii of the coaxial sample have been marked with horizontal lines. In points $\mathrm{C}$ and $\mathrm{D}$, the continuous and dashed lines represent an electron launched from the inner and outer walls, respectively.
In the range from point $C$ to point $D$, we have observed that multipactor cannot occur on the inner wall because of a lack of resonance, as depicted in Fig. 3 for point D. However, a stable single-surface discharge of order 2 can be generated on the outer metal. Thus, the significant local increase in the threshold of point $\mathrm{D}$ is again explained in terms of the variation with the radial coordinate of the applied RF electric field.

For point E, a single-surface discharge of order 2 has been found on the inner surface, which directly supposes a reduction of the RF power threshold. In this case, electron dynamics is similar to point $\mathrm{C}$.

\section{CONCLusion}

In this letter, we have studied the multipactor effect in coaxial transmission lines when an external axial dc magnetic field is applied. A significant modification of the RF power threshold as a function of the magnetic field has been observed, finding an increase and a reduction (with regard to the zero magnetic field case) of $23 \%$ and $84 \%$, respectively. Simulations performed with commercial software FEST3D have demonstrated the existence of both double- and single-sided multipactor regimes. An experiment has been designed and performed, obtaining good agreement between measurements and theoretical data.

\section{REFERENCES}

[1] J. R. M. Vaughan, "Multipactor," IEEE Trans. Electron Dev., vol. 35, no. 7, pp. 1172-1180, Jul. 1988.

[2] S. De Santis, J. M. Byrd, F. Caspers, A. Krasnykh, T. Kroyer, M. T. F. Pivi, and K. G. Sonnad, "Measurement of electron clouds in large accelerators by microwave dispersion," Phys. Rev. Lett., vol. 100, no. 9, p. 094801, Mar. 2008.

[3] R. Woo, "Multipacting discharges between coaxial electrodes," J. Appl. Phys., vol. 39, no. 3, pp. 1528-1533, Feb. 1968.

[4] E. Somersalo, P. Ylä-Oijala, and D. Proch, "Electron Multipacting in RF Structures," Hamburg, Germany, TESLA Rep. 94-14, Jul. 1994.

[5] T. P. Graves, B. LaBombard, S. Wukitch, and I. Hutchinson, "The coaxial multipactor experiment (CMX): A facility for investigating multipactor discharges," Rev. Sci. Instrum., vol. 77, no. 1, pp. 014701-1-014701-4, Jan. 2006.

[6] R. Udiljak, D. Anderson, M. Lisak, V. Semenov, and J. Puech, "Multipactor in a coaxial transmission line. Part I: Analytical study," Phys. Plasmas, vol. 14, no. 3, pp. 033508-1-033508-11, Mar. 2007.

[7] A. M. Pérez, C. Tienda, C. Vicente, S. Anza, J. Gil, B. Gimeno, and V. E. Boria, "Prediction of multipactor breakdown thresholds in coaxial transmission lines for traveling, standing, and mixed waves," IEEE Trans. Plasma Sci., vol. 37, no. 10, pp. 2031-2040, Oct. 2009.

[8] G. Becerra, Studies of Coaxial Multipactor in Presence of a Magnetic Field. Cambridge, MA: MIT, May 2007, PSFC/RR-07-6, DOE/ET54512-359.

[9] R. L. Geng, H. Padamsee, S. Belomestnykh, P. Goudket, D. M. Dykes, and R. G. Carter, "Suppression of multipacting in rectangular coupler waveguides," Nucl. Instrum. Methods Phys. Res. A, Accel. Spectrom. Detect. Assoc. Equip., vol. 508, no. 3, pp. 227-238, Aug. 2003.

[10] A. Henry and Y. Acediran, "Suppression of multipactor breakdown in satellite rectangular waveguides using DC magnetic fields," in Proc. IC $C A E$, Feb. 2010, vol. 1, pp. 766-770.

[11] FEST3D, ESA/ESTEC, Valencia, Spain, [Online]. Available: www. fest3d.com

[12] S. Anza, C. Vicente, D. Raboso, J. Gil, B. Gimeno, and V. E. Boria, "Enhanced prediction of multipaction breakdown in passive waveguide components including space charge effects," in Proc. IEEE MTT-S Microw. Symp. Dig., 2008, pp. 1095-1098.

[13] J. R. M. Vaughan, "Secondary emission formulas," IEEE Trans. Electron Dev., vol. 40, no. 4, p. 830, Apr. 1993.

[14] Multipaction Design and Test, ECSS-E-20-01A, 2003.

[15] Val Space Consortium-European Space Agency RF High-Power Laboratory, Valencia, Spain. [Online]. Available: www.val-space.com 